\title{
A RAÇA BRANCA E A FORMA HEPATOSPLENICA DA ESQUISTOSSOMOSE
}

José Tavares-Neto*

TAVARES-NETO, J. T. A raça branca e a forma hepatosplênica da esquistossomose. Rev. Saúde públ., S. Paulo, 21:342-7, 1987.

RESUMO: Foram selecionados e analisados alguns trabalhos que relataram a associação da raça branca e a forma hepatosplênica da esquistossomose mansoni. $O$ teste de Woolf, aplicado aos resultados, indicou que as amostras são homogêneas, apesar da diversidade da amostragem e, notadamente, dos critérios de classificação racial. Isto reforça a observação de que os indivíduos brancos são mais afetados pela forma hepatosplênica. Todavia, trata-se ainda de questão duvidosa, se essa associação decorre, primariamente, de um efeito biológico ou um fator social relacionado à raça.

UNITERMOS: Esquistossomose hepatosplênica, incidência. Raça caucasóide.

Vários autores relataram uma maior frequiência da forma hepatosplênica, da esquistossomose mansoni, em indivíduos com características raciais brancas (Cardoso 7 , 1953; Prata e Schroeder ${ }^{39}$, 1967; Nunesmaia e col..$^{32}$, 1975; Lira $^{26}$, 1975; Bina ${ }^{4}, 1977$; Bina e col. ${ }^{5}, 1978$; Martins-Pereira ${ }^{29}$, 1979; e Coura ${ }^{12}$, 1979). Porém, em outros relatos essa associação não foi observada (Barbosa ${ }^{3}, 1966$ e Menezes ${ }^{30}, 1976$ ).

Nos trabalhos acima citados a classificação usual foi: branco, mulato (pardo) e negro; levando em conta, principalmente, a cor da pele dos indivíduos. Portanto, a ampla variabilidade dos grupos raciais nāo foi considerada. Em alguns trabalhos retrospectivos, a classificação era realizada, muito provavelmente, por vários observadores (Cardoso", 1953; Bina e col. ${ }^{5}, 1978$; Coura ${ }^{12}$, 1979). Entretanto, Prata e Schroeder ${ }^{39}$ (1967) somente utilizaram os grupos branco e negro, com marcadas características raciais, excluindo, portanto, os mestiços." O mesmo, aparentemente, ocorreu na investigação de Cardoso? (1953). Em trabalho de Nunesmaia e col. ${ }^{32}$ (1975) o grupo branco havia sido, previamente, excluído. Já Martins-Pereira ${ }^{29}$ (1979), também, utilizou-se da classificação racial de Krieger e col. ${ }^{25}$, com uma série de inovaçōes próprias e, desse modo, não observou a associação.

Como a classificação racial baseia-se em parâmetros subjetivos e, possivelmente, variáveis entre os observadores, resolvemos testar a consistência dessa associação. Para isto, selecionamos seis trabalhos, referidos na Tabela
1, com os seguintes critérios: número de hepatosplênicos não inferior a cinco em mais de um grupo racial, referência dos dados necessários para a análise e sem critério de exclusão que distorcesse os resultados. As amostras, entre si, eram heterogêneas em suas origens, bem como nos métodos diagnósticos e critérios de classificação das formas clínicas. As formas clínicas, para efeito de análise, foram reagrupadas em dois grandes grupos: hepatointestinal (HI) e hepatosplênia (HE).

$\mathrm{Na}$ Tabela 2, verifica-se em cada amostra que a frequiência de hepatosplênicos bran$\cos$ é significantemente maior $(0,01<p$ $<0,0001)$. Aos conjuntos de dados da Tabela aplicamos o teste de Woolf ${ }^{42}$ (1955), que se mostra sensível na avaliação da significância e heterogeneidade entre amostras. Os resultados, do teste, na Tabela 3, confirmam a associação anterior, inclusive no total das amostras $\left(\mathrm{X}_{6}^{2}=110,27, \mathrm{p}<0,00001\right)$. Entretanto, a heterogeneidade entre as amostras não alcançou significância estatística $\left(X^{2}{ }_{5}=9,78\right.$, p $>$ 0,05). Portanto, apesar, das diferenças entre as amostras, os resultados foram homogêneos.

Para reforçar essa associação, raça branca e forma hepatosplênica, foram encontrados alguns relatos de correlações da doença e o alelo $\mathrm{A}$ do sistema $\mathrm{ABO}$ e dos antígenos A1/B5 do sistema de histocompatibilidade (Camus e col. ${ }^{8}, 1977$; Camus e col. ${ }^{9}$, 1979; Pereira e col. ${ }^{35}, 1979$; Salam e col. ${ }^{40}, 1979$; Kamel e col. $\left.{ }^{20}, 1984\right)$, apesar daquelas corre-

* Faculdade de Medicina do Triângulo Mineiro - Rua Getúlio Guaritá, 130 - 38100 - Uberaba, MG * Informação pessoal de Aluizio Prata. 


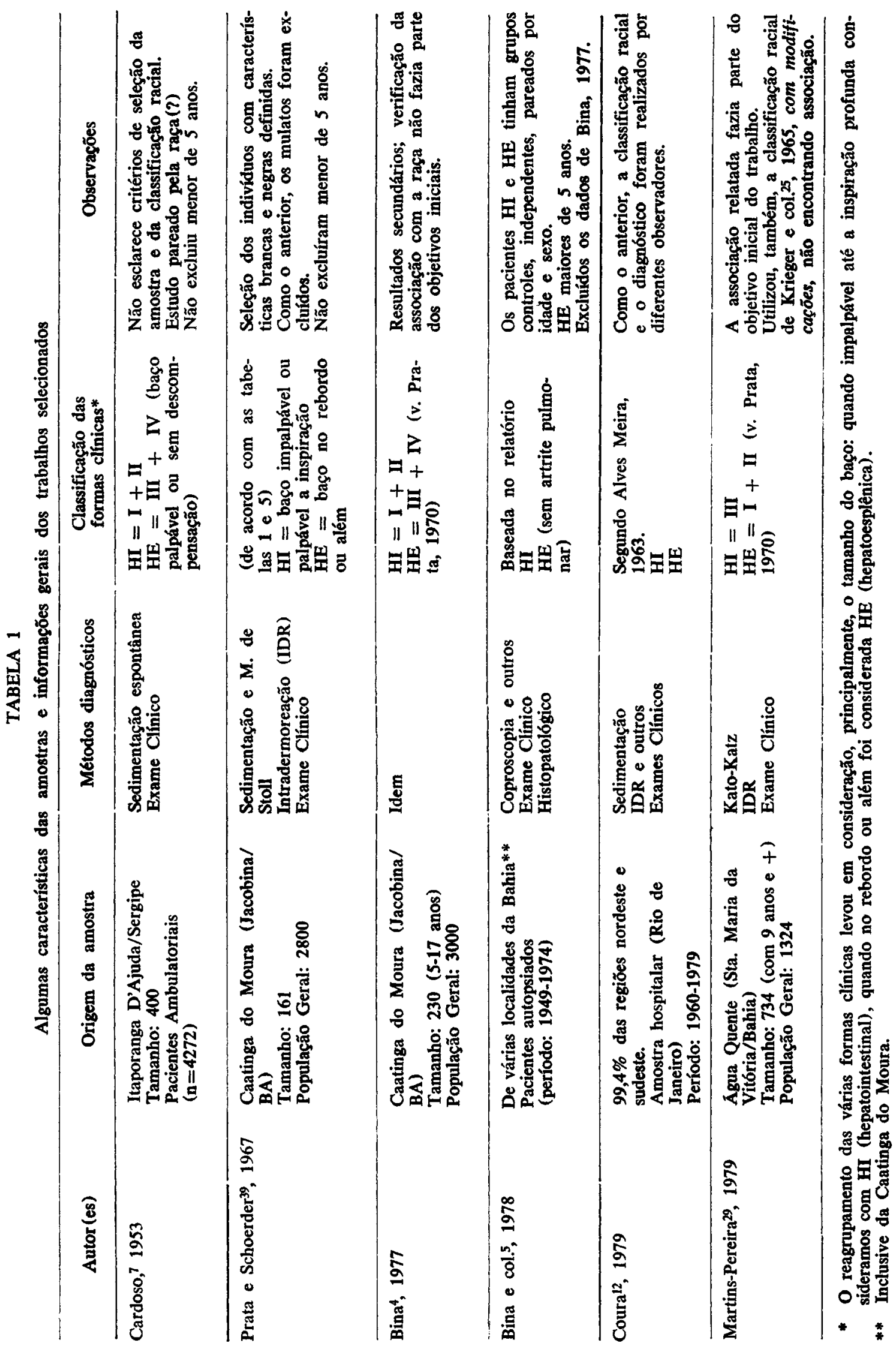


TABELA 2

Distribuição das formas clínicas da esquistossomose de acordo com os grupos raciais, em diferentes amostras brasileiras.

\begin{tabular}{|c|c|c|c|c|c|c|c|c|c|}
\hline \multirow{3}{*}{ Amostra } & \multirow{3}{*}{$\begin{array}{l}\text { Formas } \\
\text { Clínicas* }\end{array}$} & \multicolumn{6}{|c|}{ Grupos Raciais } & \multirow{3}{*}{ Total } & \multirow{3}{*}{$\begin{array}{l}\mathrm{X}^{2} \\
\mathbf{P}\end{array}$} \\
\hline & & \multicolumn{2}{|c|}{ Branco } & \multicolumn{2}{|c|}{ Mulato } & \multicolumn{2}{|c|}{ Negro } & & \\
\hline & & $\mathbf{N}$ & $\%$ & $\mathbf{N}$ & $\%$ & $\mathbf{N}$ & $\%$ & & \\
\hline Cardoso $^{7}, 1953$ & $\begin{array}{l}\mathrm{HI} \\
\mathrm{HE}\end{array}$ & $\begin{array}{r}110 \\
90\end{array}$ & $\begin{array}{l}41,0 \\
68,2\end{array}$ & - & - & $\begin{array}{r}158 \\
42\end{array}$ & $\begin{array}{l}59,0 \\
31,8\end{array}$ & $\begin{array}{l}268 \\
132\end{array}$ & $\begin{array}{c}26,05 \\
<0,0001 \\
\text { g. } 1=1 * *\end{array}$ \\
\hline $\begin{array}{l}\text { Prata e Schoerder }{ }^{39} \text {, } \\
1967\end{array}$ & $\begin{array}{l}\text { HI } \\
\text { HE }\end{array}$ & $\begin{array}{l}87 \\
22\end{array}$ & $\begin{array}{l}63,5 \\
91,7\end{array}$ & 二 & 二 & $\begin{array}{r}50 \\
2\end{array}$ & $\begin{array}{r}36,5 \\
8,3\end{array}$ & $\begin{array}{r}137 \\
24\end{array}$ & $\begin{array}{c}8,61^{* * *} \\
<0,01 \\
\mathrm{~g} .1=1\end{array}$ \\
\hline Bina 4,1977 & $\begin{array}{l}\text { HI } \\
\text { HE }\end{array}$ & $\begin{array}{r}26 \\
6\end{array}$ & $\begin{array}{l}11,9 \\
50,0\end{array}$ & $\begin{array}{r}168 \\
6\end{array}$ & $\begin{array}{l}77,1 \\
50,0\end{array}$ & 24 & $\underline{11,0}$ & $\begin{array}{r}218 \\
12\end{array}$ & $\begin{array}{c}14,27 \\
<0,001 \\
\mathrm{~g} .1=2\end{array}$ \\
\hline Bina e col.5, 1978 & $\begin{array}{l}\mathrm{HI} \\
\mathrm{HE}\end{array}$ & $\begin{array}{r}100 \\
50\end{array}$ & $\begin{array}{l}22,3 \\
34,3\end{array}$ & $\begin{array}{r}248 \\
84\end{array}$ & $\begin{array}{l}55,2 \\
57,5\end{array}$ & $\begin{array}{r}101 \\
12\end{array}$ & $\begin{array}{r}22,5 \\
8,2\end{array}$ & $\begin{array}{l}449 \\
146\end{array}$ & $\begin{array}{c}18,19 \\
<0,0001 \\
\mathrm{~g} .1=2\end{array}$ \\
\hline Coura12, 1979 & $\begin{array}{l}\text { HI } \\
\text { HE }\end{array}$ & $\begin{array}{r}1729 \\
361\end{array}$ & $\begin{array}{l}42,6 \\
60,6\end{array}$ & $\begin{array}{r}1549 \\
177\end{array}$ & $\begin{array}{l}38,2 \\
29,7\end{array}$ & $\begin{array}{r}778 \\
58\end{array}$ & $\begin{array}{r}19,2 \\
9,7\end{array}$ & $\begin{array}{r}4056 \\
596\end{array}$ & $\begin{array}{l}73,16 \\
<0,0001 \\
\text { g. } 1=2\end{array}$ \\
\hline $\begin{array}{l}\text { Martins-Pereira29, } \\
1979\end{array}$ & $\begin{array}{l}\mathrm{HI} \\
\mathrm{HE}\end{array}$ & $\begin{array}{l}76 \\
18\end{array}$ & $\begin{array}{l}11,9 \\
19,6\end{array}$ & $\begin{array}{r}368 \\
62\end{array}$ & $\begin{array}{l}57,3 \\
67,4\end{array}$ & $\begin{array}{r}198 \\
12\end{array}$ & $\begin{array}{l}30,8 \\
13,0\end{array}$ & $\begin{array}{r}642 \\
92\end{array}$ & $\begin{array}{l}14,05 \\
<0,001 \\
\text { g. } 1=2\end{array}$ \\
\hline
\end{tabular}

* HI - Hepatointestinal e HE - Hepatoesplênico;

** G.L. = graus de liberdade;

*** Com correção de Yates.

TABELA 3

Teste do Woolf aplicado aos resultados da Tabela 2

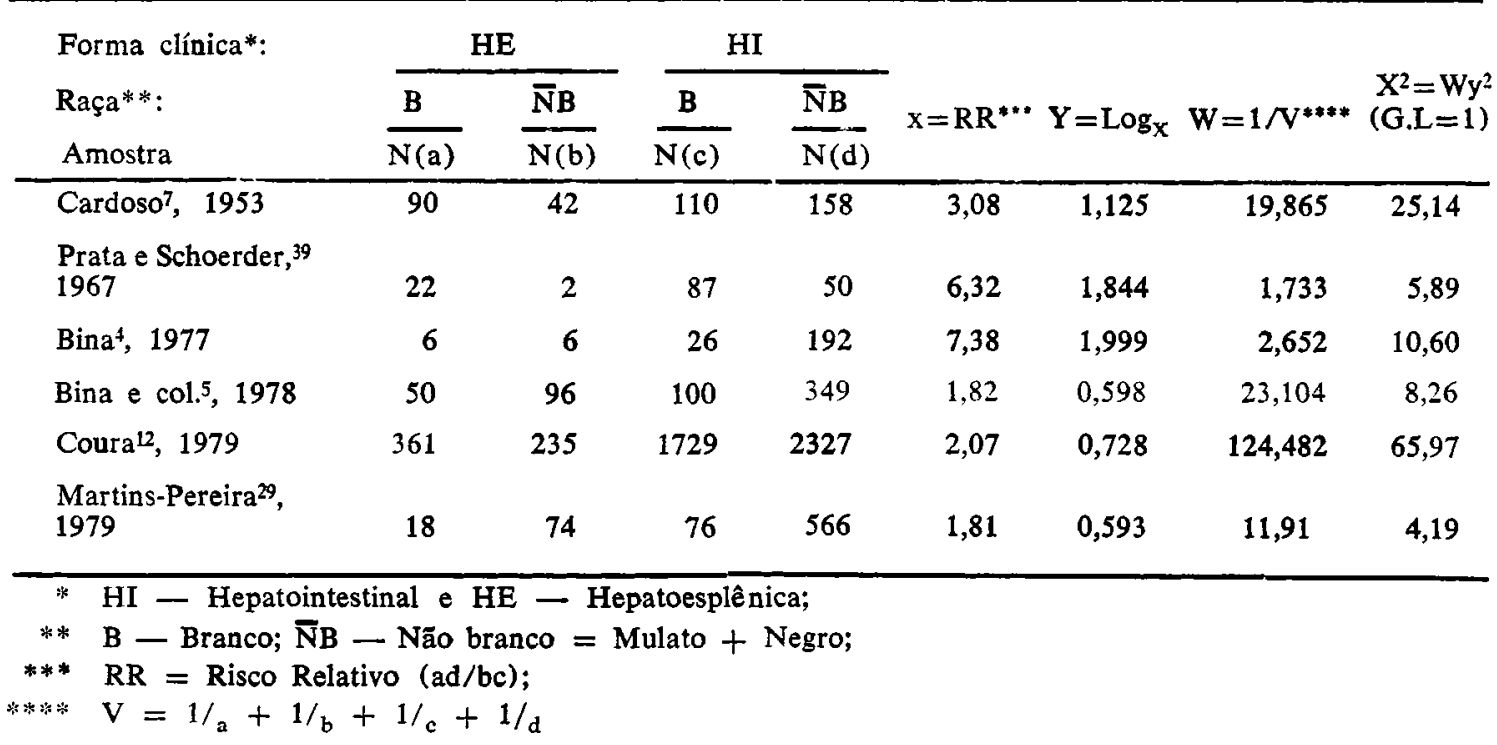

ANĂLISE DO $X^{2}$

\begin{tabular}{lrrrl}
\hline & \multicolumn{1}{|c}{$\mathbf{X}^{2}$} & G.L & \multicolumn{1}{c}{$\mathbf{P}$} \\
\cline { 2 - 2 } & & & & \\
Significância & 110,27 & 6 & $<0,00001$ \\
Heterogeneidade & 9,78 & 5 & $>0,05$ \\
\hline
\end{tabular}


lações não serem confirmadas em outras investigações (Katz e col. ${ }^{21}$, 1967; Khattab e col. ${ }^{22}$, 1968; El Masri e Sherfi ${ }^{14}, 1982$ e Martins-Pereira $^{29}$, 1979). Entretanto, esses alelos, dos sistemas ABO e HLA são mais frequientes em brancos (Arce-Gomes', 1979; Degos ${ }^{13}, 1984$ e Mourant $\left.{ }^{31}, 1954\right)$.

Por outro lado, no Brasil, os negros e mestiços vivem em piores condições socioeconômicas (Oliveira e col. ${ }^{33}, 1981$ e Silva" ${ }^{41}, 1981$ ). Assim, seria de se esperar que, entre eles, a infecção esquistossomótica fosse mais frequiente, como observaram Pellon e Teixeira ${ }^{34}$ (1950), Pessoa e Barros ${ }^{36}$ (1953) e Barbosa ${ }^{3}$ (1966). Ao contrário, Cardoso ${ }^{7}$ (1953) encontrou na população geral de Itaporanga D'Aju$\mathrm{da} /$ Sergipe mais indivíduos brancos com parasitológico positivo para o Schistosoma mansoni. Também, Pessoa e Barros ${ }^{36}$ (1953) e Barbosa $^{3}$ (1966) verificaram que a reação intradérmica, para esquistossomose, foi mais positiva entre os negróides. Já Brener e Mourão ${ }^{6}$ (1966) não observaram diferença entre os grupos raciais. Não obstante, Barbosa ${ }^{3}$ (1966) e Bina e col..$^{5}$ (1978) não encontraram diferença entre os grupos raciais e a carga parasitária do $S$. mansoni.

Assim, podemos considerar que os indivíduos, independentemente da raça, são igualmente afetados pela infecção esquistossomótica. Ou, como relatam Pellon e Teixeira ${ }^{39}$ (1950), devido à representatividade da amostragem, os negros seriam os mais infectados. Até porque, os dados de Pellon e Teixeira ${ }^{34}$ (1950) estão de acordo com a realidade socioeconômica, até atual, dos grupos negros e mestiços brasileiros (Oliveira e col. ${ }^{33}, 1981$ ).

A Africa, abaixo do Saara, onde a esquistossomose mansoni tem suas origens, as formas hapatosplênicas de esquistossomose são pouco frequientes (Gelfand ${ }^{17}, 1950$; Manson- Barh $^{28}$, 1958; Prata ${ }^{37}, 1972$ e Jordan e Weebe $\left.^{19}, 1982\right)$ e de lá vieram os negros es. cravos (Viana Filho ${ }^{42}, 1946$ e Goulart $^{18}, 1975$ ).

Deste modo, os brancos têm menos de cinco séculos de contato com a África Central e com a infecção. O que favorece a especulação de existir nos negros um fator de resistência à doença grave ou, mais propriamente, que seja mais frequiente entre eles. Azevedo ${ }^{2}$ (1984) sugeriu que a expressão desse fator esteja no fígado.

Também, pode-se especular que a resistência/susceptibilidade à forma hepatosplênica seja controlada geneticamente. Alguns indícios neste sentido, como: os dados experimentais favorecem essa hipótese (Claas e Delder ${ }^{10}$,
1979; Fanning e col. ${ }^{15}, 1981$ e Fanning e Kazura $\left.^{16}, 1984\right)$; existência em áreas de indivíduos sujeitos às mesmas condiçōes ambientais e culturais, que não evoluem para as formas graves (Bina $\left.{ }^{4}, 1977\right)$, inclusive em relação a cepa do parasito e o hospedeiro intermediário; a ocorrência maior de hepatosplênicos em certas famílias (Klöetzel ${ }^{23}$, 1958; Klöetzel $^{14}, 1962$; Conceição ${ }^{11}, 1976$; Coura ${ }^{12}, 1979$ e Prata ${ }^{38}, 1982$ ), com hábitos em relação às fontes de infecção, aparentemente, semelhantes às demais e a associação relatada com a raça branca. Mohmoud ${ }^{27}$ (1981), apreciando os trabalhos experimentais e do sistema HLA, conclui que a constituição genética do homem possa ser um dos fatores determinantes no desenvolvimento da doença.

Resta saber, porém, em relação à raça, o que é efeito biológico ou social. As raças humanas têm constituição gênica diferente. De outro lado, no Brasil, alguns indicadores socioeconômicos são diferentes entre os grupos raciais (Oliveira e col. $.^{33}, 1981$ ). Desse modo, a associação descrita não poderia, primariamente, decorrer de alguma característica ambiental ou estritamente social? Até agora, em áreas hiperendêmicas de esquistossomose, não existem evidências que brancos, negros e mestiços, por pertencerem a esse ou aquele grupo racial, comportem-se diferentemente em relação às fontes de infecção; ou com hábitos e costumes peculiares, que possam favorecer o desenvolvimento da doença grave. Muitos trabalhos de investigação objetivaram correlacionar 0 índice de infecção e a prevalência da esplenomegalia com alguns fatores de risco ou indicadores socioeconômicos, como: atividade humana, motivos de contato com águas naturais, higiene pessoal, grau de escolaridade, atividades de lazer e ocupacionais, qualidade da habitação, nível econômico, e outros. Barbosa $^{3}$ (1966), por exemplo, encontrou correlação da doença grave e precárias condiçōes de habitação, mas não com o índice da infecção. Todavia, como os indivíduos dos diferentes grupos raciais se relacionam com esses fatores? Em Catolândia e Região da Água Quente/Santa Maria da Vitória, municípios hiperendêmicos da Bahia, a forma hepatosplênica é mais frequiente nos indivíduos mais brancos. Segundo nossas observações pessoais e de forma preliminar, naquelas localidades os indivíduos brancos têm melhores condiçōes de vida e nível socioeconômico. Portanto, a questão inicial é controversa e merece investigações continuadas, para saber qual o fator de risco primário: o racial ou o racial secundário ao social. 
TAVARES-NETO, J. T. The white race and the form of hepatosphenic schistosomiasis. Rev. Saúde públ., S. Paulo, 21:342-7, 1987.

ABSTRACT: Various studies, which indicated a relation between the white race and the form of hepatosplenic schistosomiasis mansoni were selected and analyzed. Woolf's test was used, and the samples were found to be homogenous in spite of their diversity as, especially, were the racial classification criteria. This reinforces the observation that white individuals are more affected by the hepatosplenic form. It is sitll dubious, however, if this association stems primarily from a biological effect or from a social factor related to race.

UNITERMS: Schistosomiasis hepatosplenic, occurence. Caucasoid race.

\section{REFERENCIAS BIBLIOGRÁFICAS}

1. ARCE-GOMES, B. HLA: genética, variabilidade e funções biológicas. Dusenia, 11:109-19, 1979.

2. AZEVEDO, E. S. Alguns fatores genéticos na evolução da infecção pelo $S$. mansoni. In: Aspectos peculiares da infecção por Schistosoma mansoni. Salvador, CEDRE/UFBa., 1984. p. $187-99$.

3. BARBOSA, F. A. S. Morbidade da esquistossomose. Rev. bras. Malar., 18 (n. ${ }^{\circ}$ esp.): 3-159, 1966.

4. BINA, J. C. Influência da terapêutica específica na evolução da esquistossomose mansoni. Salvador, 1977. [Dissertação de Mestrado Universidade Federal da Bahia].

5. BINA, J. C.; TAVARES-NETO, J.; PRATA, A.; AZEVEDO, E. S. Greater resistence to development of severe schistosomiasis in brazilian negroes. Hum. Biol., 50:41, 1978.

6. BRENER, A. \& MOURÃO, O. G. Observações sobre a forma hepato-esplênica da esquistossomose mansoni em Minas Gerais. Rev. bras. Malar., 8:511-26, 1956.

7. CARDOSO, W. A esquistossomose mansônica no negro, Med. Cir. Farm., 202/203:89-95, 1953.

8. CAMUS, D.; BINA, J. C.; CARLIER, Y; SANTORO, F. A, B, O blood groups and clinical forms of Schistosomiasis mansoni. Trans. roy. Soc. trop. Med. Hyg., 71:182, 1977.

9. CAMUS, D.; BINA, J. C.; CARLIER, Y.; SANTORO, F.; FIGUEIREDO, J. F. M.; PRATA, A.; CAPRON, A. Grupos sangüíneos A,B,O e formas clínicas da esquistossomose mansônica. Rev. Inst. Med. trop. S. Paulo, 19:77-9, 1977.

10. CLAAS, F. H. J. \& DEELDER, A. M. H-2 linked immune response to murine experimental Schistosoma mansoni infections. $J$. Immunogenet., 6:167-75, 1979.

11. CONCEIÇÃO, M. J. Morbidade da esquistossomose mansoni em uma comunidade rural de Minas Gerais. Rio de Janeiro, 1976. [Dissertação de Mestrado - Universidade Federal do Rio de Janeiro].

12. COURA, J. R. Morbidade da esquistossomose mansoni no Brasil. Rio de Janeiro, 1979.
[Tese - Universidade Federal do Rio de Janeiro]

13. DEGOS, H. La répartion anthropologique das génes HLA et dynamique de populations. In: Cours supérieur d'hystocompatibilité. Paris, 1984. p. 131-59. [apostila].

14. El MASRI, S. H. \& SHARFI, A. R. M. A,B,O blood groups in hepatosplenic schistosomiasis. J. trop. Med. Hyg., 85:223-4, 1982.

15. FANNING, M. M.; PETERS, P. A.; DAVIS, R. S.; KAZURA, J. W. \& MAHMOUD, A. A. F. Immunopathology of murine infection with Schistosoma mansoni: relationship of genetic background to hepatosplenic disease and modulation. J. infect. Dis., 144:148-53, 1981.

16. FANNING, M. M. \& KAZURA, J. W. Genetic-linked variation in susceptibility of mice to Schistosomiasis mansoni. Parasite Immunol, 6:95-103, 1984.

17. GELFAND, M. Schistosomiasis in South Central Africa: a clinico-pathological study. Capetown, Juta Co., 1950.

18. GOULART, M. A escravidão africana no Brasil, das origens à extinção do tráfico. $3 .^{a} \mathrm{ed}$. Rio de Janeiro, Ed. Alfa-Ômega, 1975.

19. JORDAN, O. \& WEBBE, G. Epidemiology. In: Jordan, O. Schistosomiasis: epidemiology, treatment and control. William Heinemann Med. Books, 1982. p. 227-92.

20. KAMEL, M. A.; ZAKARIA, E.; MABROUK, M. A.; ZAKARIA, S.; HZAZI, A. R. M. \& El RAZIKY, E. H. HLA antigen frequencies in egyptian patients with complicated Schistosomiasis mansoni. Trans. roy. Soc. trop. Med. Hyg., 78:850, 1984.

21. KATZ, N.; TAVARES, J.; ABRANTES, W. L. $A, B, O$ and $R h$ blood groups from patients with hepatosphenic Schistosomiasis mansoni. J. Parasit., 53:99, 1967.

22. KHATTAB, M.; El-GENGEHY, M. $\Upsilon$.; SHARAF, M. A,B,O blood groups in bilharzial hepatic fibrosis. J. Egypt. med. Ass., 51:245, 1968.

23. KLOEETZEL, K. A síndrome hepatosplênica na esquistossomose mansônica: consideraçōes sobre a incidência familiar. Rev. bras. Med., 15:263-5, 1958. 
24. KLOEETZEL, K. Aspectos epidemiológicos da esquistossomose mansônica em uma população de Pernambuco. São Paulo, 1962. [Tese de Doutorado - Faculdade de Medicina da USP]

25. LIRA, L. G. C. Antígeno Austrália na esquistossomose mansônica forma hepato-esplênica. Salvador, 1975. [Tese - Universidade Federal da Bahia]

26. MAHOMOUD, A. A. F. Genetic of Schistosomiasis. In: Michal, F. Modern genetic concepts and technique in the study of parasites. London, Academic Press, 1981. p. 303-22.

27. MANSON-BARH, P. E. C. The clinical features, diagnosis and host parasite relationship of schistosomiasis in Africa. East Afr. med. J., 35:401-11, 1958.

28. MARTINS-PEREIRA, D. M. S. Sistemas HLA, $A, B, O$ e $R h$ e características raciais em pacientes com hepatoesplenomegalia esquistossomótica. Brasília, 1979. [Dissertação de Mestrado - UnB]

29. MENEZES, A. P. Esquistossomose mansônica no município de Riachuelo, Sergipe: estudo epidemiológico, clínico e laboratorial. Rio de Janeiro, 1976. [Dissertação de Mestrado UFRJ]

30. MOURANT, A. E. The distribution of the human blood groups. Boston, MA, Blackwell Scient. Publ., 1954.

31. NUNESMAIA, H. G.; AZEVEDO, E. S.; ARANDAS, E. A.; WIDMER, C. G. Composição racial e anatomoglobinomia em portadores de esquistossomose mansônica hepatoesplênica. Rev. Inst. Med. trop. S. Paulo, 17: $160-3,1975$.

32. OLIVEIRA, L. E. G. de; PORCARO, R. M.; ARAUJO, T. C. N. O lugar do negro na força de trabalho. Rio de Janeiro, FIBGE, 1981.

33. PELLON, A. B. \& TEIXEIRA, I. Distribuiçāo geográfica da esquistossomose mansônica no Brasil. Rio de Janeiro, Ministério da Educação e Saúde, 1950.

34. PEREIRA, F. E. L.; BORTOLINI, E. R.; CARNEIRO, J. L. A.; SILVA, C. R. M. da; NEVES, R. C. A,B,O blood groups and hepatoesplenic form of Schistosomiasis mansoni (Symmer's fibrosis). Trans, roy. Soc, trop. Med. Hyg., 73:238, 1979.

35. PESSOA, S. B. \& BARROS, P. R. Sobre o diagnóstico da esquistossomose mansônica na infância, pela intradermoreação com antígeno de esquistossomas adultos. Hospital, 43:19-25, 1953.

36. PRATA, A. R. I Simpósio Afro-Brasileiro sobre Esquistossomose. Brasíia méd., 2:10, 1972.

37. PRATA, A. Esquistossomose mansoni. In: Veronesi, R. Doenças infecciosas e parasitárias. São Paulo, Ed. Guanabara Koogan, 1982. p. 884-904.

38. PRATA, A, R. \& SCHROEDER, S. A comparison of whites and negroes infected with Schistosoma mansoni in a hyperendemic areas. Gaz. méd. Bahia, 67:93-8, 1967.

39. SALAM, E. A.; ISHAAC, S. \& MAHMOUD, A. A. F. Histocompatibility-linked susceptibility for hepatosplenomegaly in human Schistosomiasis mansoni. J. Immunol., 123:182931, 1979.

40. SILVA, N. V. Cor e o processo de realização sócio-econômica: dados. Rev. Cien. soc., 24: 391-409, 1981.

41. VIANNA F., L. O negro na Bahia. Rio de Janeiro, Ed. José Olympio, 1946.

42. WOOLF, B. On the estimating the relation between blood group and disease. Ann. hum. Genet., 19:251-3, 1955.

Recebido para publicação em 19/8/1986 Reapresentado em 8/5/1987

A provado para publicaçāo em 12/5/1987 